\title{
Efficacy and Tolerability of Weekly Docetaxel, Cisplatin, and 5-Fluorouracil for Locally Advanced or Metastatic Gastric Cancer Patients with ECOG Performance Scores of 1 and 2
}

\author{
Mehmet Turkeli $^{1 *}$, Mehmet Naci Aldemir ${ }^{1}$, Kerim Cayir $^{1}$, Melih Simsek ${ }^{1}$, Mehmet \\ Bilici $^{1}$, Salim Basol Tekin ${ }^{1}$, Nilgun Yildirim ${ }^{1}$, Nurhan Bilen², Ibrahim Makas ${ }^{2}$
}

\begin{abstract}
Background: Docetaxel, cisplatin, 5-fluorouracil (DCF) given every three weeks is an effective, but palliative regimen and significantly toxic especially in patients who have a low performance score. Here, we aimed to evaluate the efficacy and tolerability of a weekly formulation of DCF in locally advanced and metastatic gastric cancer patients. Materials and Methods: 64 gastric cancer patients (13 locally advanced and 51 metastatic) whose ECOG (Eastern Cooperative Oncology Group) performance status (PS) was 1-2 and who were treated with at least two cycles of weekly DCF protocol as first-line treatment were included retrospectively. The weekly DCF protocol included $25 \mathrm{mg} / \mathrm{m}^{2}$ docetaxel, $25 \mathrm{mg} / \mathrm{m}^{2}$ cisplatin, and 24 hours infusion of $750 \mathrm{mg} / \mathrm{m}^{2} 5$-fluorouracil, repeated every week. Disease and patient characteristics, prognostic factors, treatment response, grade 3-4 toxicity related to treatment, progression free survival (PFS) and overall survival (OS) were evaluated. Results: Of the patients, 41 were male and 23 were female; the median age was 63 (29-82) years. Forty-one patients were ECOG-1 and 23 were ECOG-2. Of the total, $81.2 \%$ received at least three cycles of chemotherapy. Partial response was observed in $28.1 \%$ and stabilization in $29.7 \%$. Overall, the disease was controlled in $57.8 \%$ whereas progression was noted in $42.2 \%$. The median time to progression was 4 months (95\% CI, 2.8-5.2 months) and median overall survival was 12 months (95\% CI, 9.2-14.8 months). The evaluation of patients for grade 3-4 toxicity revealed that $10.9 \%$ had anemia, $7.8 \%$ had thrombocytopenia and $10.9 \%$ had neutropenia. Non-hematologic toxicity included renal toxicity $(\mathbf{7 . 8 \%})$ and thrombosis $(1.6 \%)$. Conclusions: In patients with locally advanced or metastatic gastric cancer who were not candidates for DCF administered every-3-weeks, a weekly formulation of DCF demonstrated modest activity with minimal hematologic toxicity, suggesting that weekly DCF is a reasonable treatment option for such patients.
\end{abstract}

Keywords: Gastric cancer - locally advanced or metastatic cases - docetaxel - cisplatin - 5-fluorouracil - schedule

Asian Pac J Cancer Prev, 16 (3), 985-989

\section{Introduction}

Gastric cancer is the fourth most common cancer in the world and the second most common cause of cancerrelated death. At diagnosis, nearly two third of gastric cancer patients are in metastatic stage (Kamangar et al., 2006; Jemal et al., 2011). Systemic chemotherapy is the major treatment option at locally advanced and metastatic gastric cancer. The aim of treatment in advanced stage is palliation and improving survival. The advantage of combination treatments against single agent treatments in advanced stage gastric cancer patients is recently popular (Wagner et al., 2006).

Docetaxel, cisplatin and 5-fluorourasil (DCF) which is administered every three weeks is an effective treatment than other treatments, but it carries high toxicity risk especially in patients with poor performance status. It is required to improve optimal dose and treatment intervals to enhance the efficacy and tolerability of docetaxel based chemotherapy regimens. Phase I and phase II studies have shown that when administered weekly, docetaxel may be better tolerated (Haisworth et al., 1998; Tabernero et al., 2004). Neutropenia related to myelosupression was observed less frequent with weekly administration (Schuette et al., 2005; Camps et al., 2006).

The expected survival time is limited to 3-6 months in metastatic gastric patients who did not receive chemotherapy. This period can be prolonged to 8-12 months with platinum and taxane based chemotherapy regimens. In Tax 325 study $75 \mathrm{mg} / \mathrm{m}^{2}$ docetaxel, $75 \mathrm{mg} / \mathrm{m}^{2}$ cisplatin on D1 and $750 \mathrm{mg} / \mathrm{m}^{2}$ 5-fluorourasil on D1-D5 with 24 hours infusion was administered every three weeks in one arm and $100 \mathrm{mg} / \mathrm{m}^{2}$ cisplatin on D1 and $1000 \mathrm{mg} / \mathrm{m}^{2}$ 5-fluorourasil on D1-D5 with 24 hours infusion every 28 
days was administered in the other arm. Response rates were found as significant: $37 \%$ on DCF arm and $25 \%$ on $\mathrm{CF}$ arm. OS was also found as significant: 9.2 months on DCF arm and 8.6 months on CF arm. But grade 3-4 toxicities were observed more on DCF arm (Van Cutsem et al., 2006).

In this study, we aimed to establish the efficacy and tolerability of weekly DCF administration in locally advanced or metastatic gastric cancer patients with poor performance status by anticipating that DCF administered every three weeks may be more toxically.

\section{Materials and Methods}

Patient population: This study included retrospectively 64 gastric cancer patients (13 locally advanced and 51 metastatic) who were admitted to our hospital, Medical Oncology Department whose ECOG performance status (PS) was 1 or 2 and who were treated with at least two cycles of weekly DCF protocol as first-line treatment from February 2008 to May 2013. Patients who had received less than 2 cycles of chemotherapy were excluded. Stage IV patients had distant metastases including liver $(\mathrm{n}=33, \% 51.6)$, lung $(\mathrm{n}=7 \% 10.9)$, bone $(\mathrm{n}=2 \% 3.1)$, and peritoneum $(\mathrm{n}=19 \% 29.7)$. Four $(\% 6.3)$ patients had undergone palliative gastrectomy, 13 (\%20 .3) patients curative gastrectomy, and 47 (\%73.4) patients had no surgery. Seven (\%10.9) patients had prior adjuvant chemotherapy. The anatomic localization of gastric cancer was cardia (gastro-esophageal junction) in 38 (\%59.4) patients, body in $10(\% 15.6)$ patients, antrum in $12(\% 18.8)$ patients and diffuse in $4(\% 6.3)$ patients.

Treatment schedule and dose modification: The weekly DCF protocol included $25 \mathrm{mg} / \mathrm{m} 2$ docetaxel, $25 \mathrm{mg} / \mathrm{m} 2$ cisplatin, and 24 hours infusion of $750 \mathrm{mg} /$ m2 5-fluorouracil, repeated every week. Each cycle was repeated every 3 weeks. Fifteen (\%23.4) patients received second-line chemotherapy after weekly DCF protocol. None of the patients used prophylactic granulocyte-colony stimulating factor and antibiotics.

Assessment: Disease and patient characteristics, prognostic factors, treatment response, grade 3-4 toxicity related to treatment, progression free survival (PFS) and overall survival (OS) were evaluated. Imaging studies were documented by computed tomography and PET at baseline for every three cycles. The chemotherapy responses were evaluated according to Response Evaluation Criteria in Solid Tumors (RECIST) (Einsenhauera et al., 2009). Survival without progression was defined as the time from onset of disease to progression or death. Overall survival was defined as the time from initiation of chemotherapy to death. Performance status was defined according to the criteria of Eastern Cooperative Oncology Group (ECOG).

Statistical Analysis: All of the analyses were performed using the SPSS statistical software program package (SPSS version 20.0 for windows). Progression free survival and overall survival were calculated with the logrank test. The Kaplan-Meier method was used for survival curves. Differences were assumed to be significant when $\mathrm{p}$ value was less than 0.05 .

\section{Results}

\section{Patient characteristics}

Of the patients, 41 were male and 23 were female; the median age was 63 (29-82) years. 41 patients were ECOG1 and 23 patients were ECOG-2. Of all patients, $81.2 \%$ received at least three cycles of chemotherapy and $51.6 \%$ completed the preplanned six cycles of treatment. Twelve (18.8\%) patients received 2 cycles, 19 (29.7\%) patients received 3 cycles, $32(50 \%)$ patients received 6 cycles and $1(1.6 \%)$ patient received 9 cycles of chemotherapy. Complete response was not obtained in any patients. The patient characteristics are listed in Table 1.

Among seventeen variables of univariate analysis, four variables were identified to have prognostic significance: disease status $(\mathrm{p}=0.02)$, prior curative or palliative surgery $(\mathrm{p}=0.03)$, prior chemotherapy $(\mathrm{p}=0.01)$, and absence of peritoneum metastasis $(\mathrm{p}=0.04)$ (Table 2). Multivariate analysis by Cox proportional hazard model which included these four prognostic significance factors showed that prior chemotherapy was considered independent prognostic factors for survival (OR:3.86, 95\%CI: 1.07$13.95, \mathrm{p}=0.039$ ).

\section{Efficacy}

Partial response was observed in 18 patients $(28.1 \%)$ and stabilized disease was observed in 19 patients $(29.7 \%)$; overall, the disease was controlled in 37 patients $(57.8 \%)$ whereas progression was observed in 27 patients (42.2\%). The median follow-up time was 8 months (min-max: 2-66 months), median time to progression was 4 months (95\%CI: 2.8-5.2 months) and median overall survival was 12 months (95\%CI: 9.2-14.8 months). These are shown in

Table 1. The General Characteristics of The Patients

\begin{tabular}{|c|c|c|}
\hline Characteristic & $\begin{array}{l}\text { No. of patients } \\
\qquad(n=64)\end{array}$ & $\%$ \\
\hline \multicolumn{3}{|l|}{ Age (years) } \\
\hline Median (Range) & $63(29-82)$ & \\
\hline Male & 41 & 64.1 \\
\hline \multicolumn{3}{|l|}{ ECOG performance status } \\
\hline 1 & 41 & 64.1 \\
\hline 2 & 23 & 35.9 \\
\hline \multicolumn{3}{|l|}{ Disease status } \\
\hline Locally advanced & 13 & 20.3 \\
\hline Metastatic & 51 & 79.7 \\
\hline \multicolumn{3}{|l|}{ Anatomic site } \\
\hline Cardia & 38 & 59.4 \\
\hline Body & 10 & 15.6 \\
\hline Antrum & 12 & 18.8 \\
\hline Diffuse & 4 & 6.3 \\
\hline \multicolumn{3}{|l|}{ Prior surgery } \\
\hline None & 47 & 73.4 \\
\hline Curative gastrectomy & 13 & 20.3 \\
\hline Palliative gastrectomy & 4 & 6.3 \\
\hline Prior chemotherapy & 7 & 10.9 \\
\hline \multicolumn{3}{|l|}{ Metastatic sites } \\
\hline Liver & 33 & 51.6 \\
\hline Lung & 7 & 10.9 \\
\hline Bone & 2 & 3.1 \\
\hline Peritoneum & 19 & 29.7 \\
\hline Dose reduction & 4 & 6.3 \\
\hline Second-line chemotherapy & 15 & 23.4 \\
\hline
\end{tabular}


Table 2. Univariate Analysis of Survival Time by Categorical Variable

\begin{tabular}{lll}
\hline Response & Log-rank test value $\mathrm{p}$ \\
\hline Age & 0.001 & 0.9 \\
Gender & 0.1 & 0.7 \\
Stage & 0.7 & 0.4 \\
Performance status & 0.004 & 0.9 \\
Disease status (local advanced/metastatic) & 5.2 & 0.02 \\
Anatomic site & 0.5 & 0.5 \\
Prior surgery & 2.4 & 0.1 \\
Prior curative or palliative surgery & 4.5 & 0.03 \\
Prior chemotherapy & 6.1 & 0.01 \\
Dose reduction & 0.2 & 0.67 \\
Second line chemotherapy & 1.5 & 0.2 \\
Weight loss & 0.004 & 0.95 \\
Hypertension & 0.7 & 0.4 \\
Liver metastasis & 0.4 & 0.5 \\
Lung metastasis & 0.2 & 0.6 \\
Bone metastasis & 0.06 & 0.8 \\
Peritoneum metastasis & 4.2 & 0.04 \\
\hline
\end{tabular}

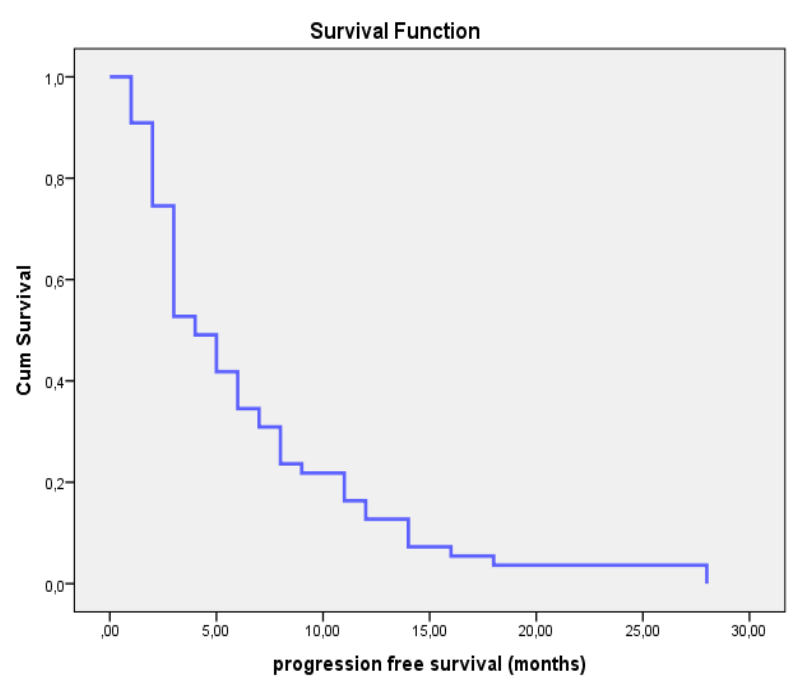

Figure 1. Progression-free Survival for All Treated Patients

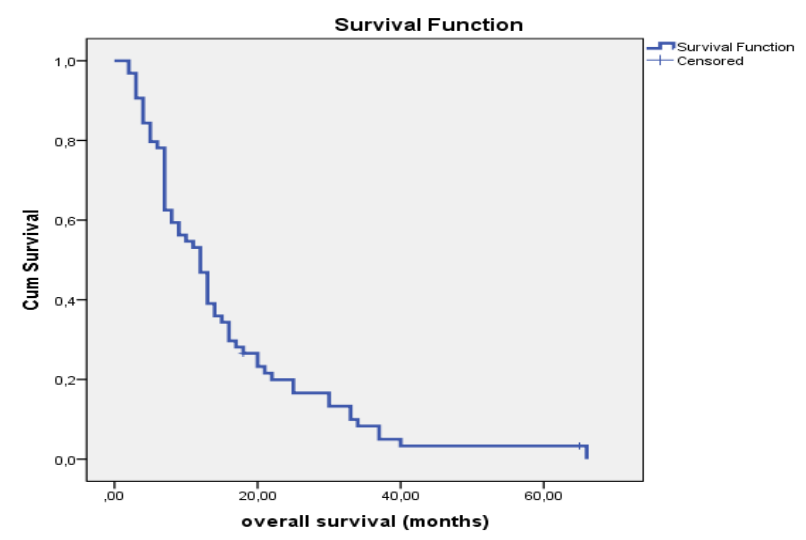

Figure 2. Overall Survival for All Treated Patients

Figure 1 and 2. The 1-year survival rate for patients was $47 \%$, with an estimated 2-year survival rate of $17 \%$. One of the patients had 5 years survival.

Adverse Reactions: The hematologic toxicity profile is summarized in Table 3. Non-hematologic toxicities
Table 3. Grade 3-4 Hematologic and Non-hematologic Toxicities (National Cancer Institute Common Toxicity Criteria, Version 3.0)

\begin{tabular}{lcr}
\hline Grade 3-4 toxicity & $\begin{array}{c}\text { No. of patients } \\
(\mathrm{n}=64)\end{array}$ & $\%$ \\
\hline Hematologic toxicity & & \\
$\quad$ Neutropenia & 7 & 10.9 \\
Anemia & 7 & 10.9 \\
Febrile neutropenia & 1 & 1.6 \\
Thrombocytopenia & 5 & 7.8 \\
Non-hematologic toxicity & & \\
$\quad$ Renal toxicity & 5 & 7.8 \\
Thrombosis & 1 & 1.6 \\
\hline
\end{tabular}

including nausea, vomiting, diarrhea, sensorial neuropathy, mucositis, hepatic toxicity and allergic reactions were not observed (Table 3 ). None of the patients died due to chemotherapy toxicity.

\section{Discussion}

With this study, we showed that in patients with locally advanced or metastatic gastric cancer who were not candidates for DCF that were administered every-3weeks, a weekly formulation of DCF demonstrated modest activity with minimal hematologic toxicity, suggesting that weekly DCF is a reasonable treatment option for such patients.

DCF regimen is the first-line regimen that is used frequently in metastatic gastric cancer despite its high toxicity and low tolerability. DCF which is administered every three weeks is an effective treatment than other treatments, but is a palliative regimen with high toxicity risk especially in patients with poor performance status. Phase I and phase II studies have shown that when administered weekly, tolerability of docetaxel is better (Haisworth et al., 1998; Tabernero et al., 2004). Neutropenia related to myelosupression was observed less frequent with weekly administration (Schuette et al., 2005; Camps et al., 2006).

In Tax 325 study; $75 \mathrm{mg} / \mathrm{m}^{2}$ docetaxel, $75 \mathrm{mg} / \mathrm{m}^{2}$ cisplatin on D1 and $750 \mathrm{mg} / \mathrm{m}^{2}$ 5-fluorourasil on D1-D5 with 24 hours infusion was administered every three weeks in one arm and $100 \mathrm{mg} / \mathrm{m}^{2}$ cisplatin on D1 and $1000 \mathrm{mg} / \mathrm{m} 2$ 5-fluorourasil on D1-D5 with 24 hours infusion every 28 days was administered in the other arm. Response rates were found as $37 \%$ on DCF arm and $25 \%$ on CF arm. Median OS was found as 9.2 months on DCF arm and 8.6 months on CF arm. But grade 3-4 toxicities were observed more on DCF arm (Van Cutsem et al., 2006)

In a phase I study which was performed by Wang et al. in metastatic gastric cancer patients, a chemotherapy including docetaxel $33 \mathrm{mg} / \mathrm{m}^{2}$, cisplatin $30 \mathrm{mg} / \mathrm{m}^{2}$ and 5-fluorourasil 1000-1750 mg/m² D1, D8, D15 was administered every 28 days. In conclusion of the study dosage of 5-FU was evaluated. The most effective and most tolerable dose was recommended as docetaxel 33 $\mathrm{mg} / \mathrm{m}^{2}$, cisplatin $30 \mathrm{mg} / \mathrm{m}^{2}$ and 5-fluorourasil $1500 \mathrm{mg} / \mathrm{m} 2$ D1, D8, D15 every 28 days (Wang et al., 2008).

In a meta-analysis performed by Chen et al. (2013) 
which was about DCF administered palliatively in gastric cancer, DCF and non-taxane chemotherapy regimens were compared. In DCF arm, response rates were found as complete response \%5.0, partial response \%38.8, stable disease $\% 27.5$ and these response rates were higher than the response rates in our study. In Tax 325 study response rates were found as complete response $2 \%$, partial response $35 \%$, stable disease $30 \%$ in DCF administered arm and these results were better than our study results.

In our study, docetaxel was administered with standard DCF dose and cisplatin was divided into three doses and 5 -FU was administered with a dose of $750 \mathrm{mg} / \mathrm{m}^{2} /$ day 24 hours infusion to decrease toxicity; the cycle was repeated every 21 days. Complete response was not obtained in any patients. Partial response was observed in $28.1 \%$ and stabilized disease was observed in $29.7 \%$. Overall, the disease was controlled in $57.8 \%$ whereas progression was observed in $42.2 \%$. These results may depend on lack of no ECOG PS-0 patients in our study.

The median follow-up time was 8 months (min-max: 2-66 months), median time to progression was 4 months (95\% CI, 2.8-5.2 months) and median overall survival time was 12 months (95\% CI, 9.2-14.8 months). The 1-year survival rate for patients was $47 \%$, with an estimated 2-year survival rate of $17 \%$. One of the patients had 5 years survival. In a meta-analysis performed by Chen and al. which was about DCF administered palliatively in gastric cancer and included 9 studies on DCF arm PFS was found as 4.6-6.8 months and OS was found as 9.012.0 months. In Tax 325 study on DCF administered arm PFS was found as 5.6 months and OS was found as 9.2 months. In our study although PFS was shorter, OS was found similar to the best response rates. (Chen et al., 2013; Van Cutsem et al., 2006).

In Tax 325 study, grade 3-4 toxicity rates were: neutropenia $82 \%$, anemia $18 \%$, thrombocytopenia $8 \%$, febrile neutropenia $29 \%$, nausea $14 \%$, vomiting $17 \%$ and diarrhea (19\%). In meta-analysis performed by Chen et al. (2013) toxicity evaluation on DCF arm revealed grade 3-4 neutropenia rate as $61.3 \%$, febrile neutropenia rate as $23.3 \%$, anemia rate as $14.2 \%$ and these rates were higher than our study; conversely, thrombocytopenia rate was $5.1 \%$ and was found lower. In evaluation of grade 3-4 non-hematologic toxicities while diarrhea $15.2 \%$, nauseavomiting $13.1 \%$, mucositis $16.8 \%$, neuropathy $6.5 \%$ were observed higher compared to our results. In our study, grade 3-4 toxicity rates (neutropenia $10.9 \%$, anemia $10.9 \%$ and thrombocytopenia $7.8 \%$ ) were lower than the Tax 325 study. Non-hematologic toxicities including nausea, vomiting, diarrhea, sensorial neuropathy, mucositis, hepatic toxicity and allergic reactions were not observed, but five patients had renal toxicity $(7.8 \%)$ and one patient had deep vein thrombosis $(1.6 \%)$.

Ozdemir et al. (2010) have found that have comparable efficacy with classical DFC, and also had better toxicity profile. In the reduced-dose DCF arm, complete response $5.4 \%$, partial response $21.6 \%$, stable disease $37.9 \%$, progressive disease $13.5 \%$ and overall response $64.9 \%$ were similar in both groups. Grade 3-4 toxicity revealed that $8.1 \%$ neutropenia, $5.4 \%$ anemia, $5.4 \%$ nausea, and $\% 5.4$ diarrhea were much lower than classical DCF arm
(Ozdemir et al., 2010).

Alici et al. have found that low-dose DC- De Gramont regimen is active in metastatic gastric cancer with a tolerable toxicity profile. Chemotherapy-naive patients with metastatic gastric cancer received docetaxel 60 $\mathrm{mg} / \mathrm{m}^{2}$ on day 1 and cisplatin $30 \mathrm{mg} / \mathrm{m}^{2}$ on day $1-2$ and the De Gramont regimen (Folinic acid $400 \mathrm{mg} / \mathrm{m}^{2}$ on day 1 and 5 -FU $2400 \mathrm{mg} / \mathrm{m}^{2} / 46 \mathrm{~h}$ continuous infusion) every 3 weeks. The overall response rate was $56.6 \%$. Stable disease was observed in $23.3 \%$ and progression occurred in $43.3 \%$. The median time to progression was 7 months (95\%CI 6-7.9). The median overall survival time was 15 months (95\%CI 13.7-16.2). The most frequent hematological toxicity was leucopenia, which occurred at grade 3/4 intensity in 20\% (Alici et al., 2013).

In our study, among seventeen variables of univariate analysis, four variables were identified to have prognostic significance: disease status to be local advanced $(\mathrm{p}=0.02)$, prior curative surgery $(\mathrm{p}=0.03)$, prior chemotherapy $(\mathrm{p}=0.01)$, and absence of peritoneum metastasis $(\mathrm{p}=0.04)$. Multivariate analysis which included four prognostic significant factors showed that prior chemotherapy was considered an independent prognostic factor for survival. Absence of peritoneum metastasis was found as a significant prognostic factor similar with the studies by Kim et al., 2008 and Inal et al., 2012.

In a retrospective study performed by İnal et al., 107 locally advanced or metastatic gastric cancer patients who were treated with DCF as first-line treatment were analyzed. Performance status, weight loss, histology, peritoneum metastasis, ascites and serum hemoglobin levels were identified as important prognostic factors in advanced gastric cancer patients. These findings may facilitate pretreatment prediction of survival and can be used for selecting patients for treatment (Inal et al., 2012).

Limitations about our study was, had no control group, to be a retrospective study, single center included and small size of patients. Also weekly DCF administration may be not cost-effective. They need to be investigated.

In conclusion, in patients with ECOG performance status 1-2, weekly DCF administration may be used. It has similar OS results compared to literature while median PFS times seems lower. Toxicity seems to be more tolerable. Weekly divided DCF may be an alternative regimen especially in patients with ECOG PS 1-2. On this account, larger and prospective studies are required about weekly DCF subject.

\section{References}

Alici S, Buyukberber S, Alkis N, et al (2013). Low-dose docetaxel/cisplatin - leucovorin and 46 hour infusional fluorouracil in metastatic gastric carcinoma. Anatolian Society of Medical Oncology. Asian Pac J Cancer Prev, 14, 423-7.

Eisenhauera EA, Therasseb P, Bogaertsc J, et al (2009). New response evaluation criteria in solid tumours: Revised RECIST guideline (version 1.1) Eur J Cancer, 45, 228-47

Camps C, Massuti B, Jimenez A, et al (2006). Randomized phase III study of 3-weekly versus weekly docetaxel in pretreated advanced non-small-cell lung cancer: a Spanish Lung Cancer Group trial. Ann Oncol, 17, 467-72. 
Chen XL1, Chen XZ, Yang C, et al (2013). Docetaxel, cisplatin and fluorouracil (DCF) regimen compared with non-taxanecontaining palliative chemotherapy for gastric carcinoma: a systematic review and meta-analysis. PLoS One. 8, 60320

Jemal A, Bray F, Center MM, et al (2011). Global cancer statistics. CA Cancer J Clin, 61, 69-90.

Hainsworth JD, Howard AB III, Erland JB et al (1998). Phase I trial of docetaxel administered by weekly infusion in patients with advanced refractory cancer. J Clin Oncol, 16, 2164-8.

Inal A, Kaplan MA, Kuçukoner M, et al (2012). Prognostic factors in first-line chemotherapy treated metastatic gastric cancer patients: a retrospective study. Asian Pac J Cancer Prev, 13, 3869-72.

Kamangar F, Dores GM,Anderson WF (2006). Patterns of cancer incidence, mortality, and prevalence across five continents: defining priorities to reduce cancer disparities in different geographic regions of the world. J Clin Oncol, 24, 2137-50.

Kim JG, Ryoo BY, Park YH, et al (2008). Prognostic factors for survival of patients with advanced gastric cancer treated with cisplatin-based chemotherapy. Cancer Chemother Pharmacol, 61, 301-7

Ozdemir NY, Abali H, Oksüzoğlu B, et al (2010). The efficacy and safety of reduced-dose docetaxel, cisplatin, and 5-fluorouracil in the first-line treatment of advanced stage gastric adenocarcinoma. Med Oncol, 27, 680-4.

Schuette W, Nagel S, Blankenburg T, et al (2005). Phase III study of second-line chemotherapy for advanced non-small-cell lung cancer with weekly compared with 3-weekly docetaxel. J Clin Oncol, 23, 8389-95

Tabernero J, Climent MA, Lluch A et al (2004). A multicentre, randomised phase II study of weekly or 3-weekly docetaxel in patients with metastatic breast cancer. Ann Oncol, 15, 1358-65.

Van Cutsem E, Moiseyenko VM, Tjulandin SA, Majlis A, Constenla M et al (2006). V325 Study Group Phase III study of docetaxel and cisplatin plus fluorouracil compared with cisplatin and fluorouracil as first-line therapy for advanced gastric cancer: a report of the V325 study group. J Clin Oncol, 24, 4991-7.

Wang B, Zhang W, Hong X, Guo Y, Li J (2009). Phase I doseescalating study of $24 \mathrm{~h}$ continuous infusion of 5 -fluorouracil in combination with weekly docetaxel and cisplatin in patients with advanced gastric cancer. Cancer Chemother Pharmacol. 63, 213-8.

Wagner AD, Grothe W, Haerting J, et al (2006). Chemotherapy in advanced gastric cancer: a systematic review and metaanalysis based on aggregate data. JCO, 24, 2903-9 